\section{Capacitaciones en huertos caseros, una alternativa de producción familiar para la etnia Saraguro al sur del Ecuador}

\author{
Natacha Fierro \\ ndfierro@utpl.edu.ec \\ Daniel Capa Mora
}

edcapa@utpl.edu.ec

Luis Jaramillo

luis.vicnet@hotmail.com

\author{
Leticia Jiménez \\ Isjimenez@utpl.edu.ec \\ Universidad Técnica Particular \\ de Loja, Ecuador.
}

Investigación y extensión universitaria / Intervenciones
RECEPCIÓN: 03/05/18

ACEPTACIÓN FINAL: 28/06/18

\begin{abstract}
Resumen
La presente investigación evaluó conocimientos y capacidades técnicas antes y después de un programa de formación sobre producción de huertos familiares, el cual fue dirigido por docentes y estudiantes de la Universidad Técnica Particular de Loja como aporte a la seguridad alimentaria en Ecuador, provincia de Loja, cantón Saraguro. El currículo de capacitaciones consistió en la implementación y manejo de huertos familiares dirigido a 40 familias indígenas del Consejo de Sanadores Comunitarios de Saraguro, que incluyen grupos de Mamas y Taitas Yachaks, como sobadores, yerbateros, parteras, visionarios y agricultores que emplean plantas medicinales y costumbres ancestrales. Se aplicó la metodología de Escuela de Campo. Los resultados fueron alentadores para los participantes, ya que se contribuyó a mejorar de manera sostenible la producción en sus huertos y visualizó el interés de los grupos en fortalecer sus capacidades productivas que permita disponer de materia prima para sus prácticas de medicina ancestral, autoconsumo y sustento familiar.
\end{abstract}

Palabras clave: capacitación, intervención comunitaria, huertos familiares, sanadores
Training in home gardens, an alternative family production for the Saraguro ethnic group in southern Ecuador

\begin{abstract}
This research evaluated knowledge and technical skills before and after a training program on production of family gardens, this was led by teachers and students of the UTPL as a contribution to food security in Ecuador, province of Loja, Saraguro canton. The training curriculum consisted in the implementation and management of family vegetable gardens for 40 indigenous families of the Community Healers Council of Saraguro, which includes groups of Mamas and Taitas Yachaks, such as sobadores, yerbateros, midwives, visionaries and farmers who use medicinal plants and ancestral customs. The methodology of Escuela de Campo was applied. The results were encouraging for the participants, since they contributed to the sustainable improvement of production in their gardens; visualizing the interest of the groups in strengthening their productive capacities that allow to have raw material for their practices of ancestral medicine, self-consumption and family sustenance.
\end{abstract}

Keywords: training, community intervention, homegardens, healers
Treinamento em hortas caseiras, uma produção familiar alternativa para a etnia Saraguro no sul do Equador

\section{Resumo}

Esta pesquisa avaliou conhecimentos e habilidades técnicas antes e depois de um programa de treinamento sobre a produção de hortas familiares; ele foi conduzido por professores e alunos da Universidade Técnica de Loja como uma contribuição para a segurança alimentar no Equador, província de Loja, cantão Saraguro. O currículo de formação se baseou na implementação e gestão de hortas familiares, destinado a 40 famílias indígenas do Conselho de Curadores Comunitário de Saraguro, que incluem grupos de Mamas e Taitas Yachaks como sovadores, ervateiros, parteiras, visionários e agricultores que utilizam plantas medicinais e costumes dos ancestrais. Utilizou-se a metodologia da Escola de Campo. Os resultados foram animadores para os participantes, pois contribuíram para a melhoria sustentável da produção em suas hortas; visualizando o interesse dos grupos em fortalecer suas capacidades produtivas que permita dispor da matéria-prima para suas práticas de medicina dos ancestrais, autoconsumo e sustento familiar.

Palavras-chave: treinamento, intervenção comunitária, hortas familiares, curadores

Para citación de este artículo: Fierro, N.; Capa Mora, D.; Jaramillo, L. y Jiménez, L. (2018). Capacitaciones en huertos caseros, una alternativa de producción familiar para la etnia Saraguro al sur del Ecuador. +E: Revista de Extensión Universitaria, 8(8), enero-junio, 174-186. doi: 10.14409/extensión. v8i8.Ene-Jun.7726. 


\section{Introducción}

En todo el mundo, y por supuesto en Latinomérica y, más precisamente en Ecuador, las actividades agrícolas del sector rural son muy significativas para el desarrollo de un país, y es por ello que se ha visto factible la revalorización de este sector. La zona rural es el centro esencial desde el punto de vista de la agricultura, ya que esta es su actividad principal, con la que se construyen sociedades, institucionalidad, cadenas productivas y asentamientos de diversas culturas (Núñez, 2004).

La superficie de uso del suelo y la producción agropecuaria juegan un papel importante dentro de la economía del país, representan un $8 \%$ del producto interno bruto, con un crecimiento interanual del $4 \%$ (Monteros y Salvador, 2015). La superficie en Ecuador para uso agrícola de los cultivos transitorios y barbechos en el año 2015 fue 951000 hectáreas que equivalieron al 16,76 \%; asimismo, la superficie de labor agropecuaria en la provincia de Loja comprendió una participación nacional del 4,80 \% y la producción destinada a los cultivos transitorios en el año 2015 tuvo una superficie de 49494 ha, que representó el 0,38 \% (ESPAC, 2015). Según Cueva y Chalán (2010), la cobertura de cultivos asociados andinos en el cantón Saraguro es de 2456 ha, correspondiente al 2,31 \% y los cultivos asociados subtropicales constan de una superficie de 2085 ha, equivalente al 1,96 \%.

La producción de cultivos transitorios $u$ hortalizas es indispensable para una dieta balanceada, la cual debe proporcionar al cuerpo diversos nutrientes, energía, vitaminas, minerales y antioxidantes, necesarios para proteger la salud, prevenir enfermedades y apoyar a la seguridad alimentaria (Ministerio de Salud, 2016). Consecuentemente, el uso de agroquímicos sintéticos produce un efecto negativo en la salud y además ocasiona daños al medio ambiente; es por ello que las buenas prácticas en agricultura amigable con el ambiente son de vital relevancia para los pequeños y medianos productores, puesto que pueden mejorar la calidad de vida y además generar ingresos. Es necesario integrar el conocimiento local con el conocimiento científico para promover la participación horizontal, intercambiar experiencias de manera holística, rescatar saberes primigenios que trae el campesino arraigado a su trascendental contacto con la tierra con miras a lograr el desarrollo rural sostenible (López et al., 2008).

La capacitación es un proceso que debe estar presente en toda actividad rural. Bajo los paradigmas o acción por los que se adquiere aptitud y habilidad para realizar algo con la suficiente humildad y capacidad para considerar y aprovechar la experiencia y conocimientos que los demás tienen, a través de diferentes eventos de formación, los saberes de los campesinos se transmiten a toda la comunidad rural (IICA, 1992). Además, la capacitación o educación no formal por medio de la asesoría permite satisfacer necesidades sobre la base de competencias que demandan las comunidades, enfocadas al conocimiento, habilidades y actitudes de sus miembros en sus procesos de cambio, crecimiento y adaptación a nuevas circunstancias internas y externas para integrar la información técnica con los saberes locales, fortalecer y construir nuevo conocimiento a ser puesto en práctica (Blake, 1997; FAO, 2011).

El trabajo de ampliar las investigaciones científicas y conocimientos técnicos en prácticas agrícolas se realiza a través de la educación para promover la aceptación de los conocimientos con relación a una producción eficiente. Por tal motivo, es importante la difusión mediante capacitaciones que faciliten la intermediación y el intercambio de conocimientos, 
y esta intermediación se efectúa con procesos de asesoría, extensión e intervención comunitaria (FAOa, 2010).

Uno de los principales objetivos de la asesoría e intervención comunitaria es fortalecer lazos sociales, generar compromiso con el grupo y la organización, y mantener reuniones que respaldan el conocimiento ancestral e intercultural al transmitir información valedera que preserve las costumbres y tradiciones tanto en el ámbito social como productivo (Suárez, 2000).

Muchos de los pueblos y comunidades indígenas conservan ciertas costumbres, prácticas y saberes reconocidos durante décadas entre sus generaciones de manera milenaria, entre ellos, la medicina ancestral, que complementa el bienestar corporal con lo espiritual haciendo uso de plantas y yerbas en sus rituales y remedios. Pacheco menciona (como se citó en Marín, 2017): "Los saberes de la curación ancestral son el producto de la sociedad y sus relaciones con el medioambiente, la naturaleza y la concepción íntima de la persona o paciente, para estar en comunión con la naturaleza".

La Organización Mundial de la Salud (OMS), en su Programa de Medicina Tradicional, define las prácticas ancestrales como enfoques, conocimientos y creencias diversas que son incorporadas en sus rituales, medicinas brebajes, terapias espirituales y técnicas manuales sobre la base de plantas, animales y/o minerales, aplicados ya sea de manera individual o de forma combinada para prevenir, diagnosticar, tratar enfermedades y mantener el bienestar (OMS, 2002). La misma organización, en 2013, señaló que la medicina ancestral incluye conocimientos, aptitudes y prácticas fundamentadas en teorías, creencias y experiencias indígenas de las diferentes culturas, sean o no explicables, puestas en práctica a favor de la salud y el bienestar, así como para la prevención, el diagnóstico, la mejora o el tratamiento de enfermedades tanto de la mente como del cuerpo y el espíritu.

Al sur del Ecuador se encuentra el cantón Saraguro; sus pobladores, de nacionalidad indígena, son llamados "Saraguros" y desarrollan importantes prácticas ancestrales. Para ello cuentan con el Consejo de Sanadores Ancestrales Comunitarios Hampiyachakkuna, integrado por 42 familias, de las cuales el $64 \%$ representa a 27 miembros pertenecientes al sexo masculino y jefes de hogar; el $36 \%$, un total de 15 miembros, corresponde al sexo femenino y son jefas de hogar, lo que significa que la participación de la mujer es alta y su aporte significativo para lograr el sustento del hogar (Jaramillo, 2016). Los Yachakkuna (curanderas tradicionales saraguras o Yachakkuna o Mama Yachak) (Marín Pérez et al., 2016) usan especies psicoactivas como el cactus San Pedro (Echinopsis pachanoi), el wandug (Brugmansia spp.), el tabaco (Nicotiana tabacum L.) en sus rituales mágico-religiosos y ceremonias curativas para tratar enfermedades físicas, mentales, emocionales y sobrenaturales (Armijos, Cota y González, 2014).

Conscientes de la importancia de ejecutar proyectos de desarrollo rural, en el año 2016, la Universidad Técnica Particular de Loja (UTPL) y la Dirección Provincial de Salud de Loja realizaron un trabajo de investigación junto con el grupo de Sanadores Ancestrales Comunitarios Hampiyachakkuna, quienes promueven los conocimientos y saberes ancestrales para mantener el equilibrio entre el hombre y la naturaleza y realizaron un primer estudio etnobotánico sobre el uso de especies medicinales. En dicho estudio se reportaron 174 especies y sus resultados se presentan en el libro Especies medicinales silvestres empleadas por la etnia Saraguro en la parroquia San Lucas, de los autores Andrade, Armijos, Malagón y Lu- 
cero (2009). Sin embargo, no basta con conocer las propiedades curativas, uso y beneficio de las plantas, sino que se requiere de la disposición de las mismas y con facilidad en cada uno de los hogares, ya que son materia prima básica en sus rituales y tratamientos. Es por ello, que en reuniones de trabajo se expuso la necesidad formativa y de capacitación técnica en lo que respecta al manejo de huertos familiares para el cultivo de hortalizas, verduras y plantas medicinales.

En el presente proyecto productivo partimos, entonces, de un currículo de capacitaciones teórico-prácticas basadas en las necesidades, problemas y recursos locales en las cuales se emplearon herramientas participativas como talleres temáticos y la Escuela de Campo (ECA), apoyados en la educación no formal y enfocados en el manejo tecnificado de huertos caseros dirigido al Consejo de Sanadores Comunitario del cantón Saraguro, con el objeto de fortalecer sus capacidades productivas y disponer de materia prima para sus prácticas de medicina ancestral, incrementar la producción de sus cultivos, contribuir a una alimentación básica, mejorar los ingresos económicos y contribuir así a la soberanía y seguridad alimentaria.

\section{Metodología de abordaje de nuestro estudio}

Esta investigación fue parte del "Programa de Responsabilidad Social Universitaria con el Consejo de Sanadores Comunitario de Saraguro", desarrollado en el cantón Saraguro, el cual está ubicado al norte de la provincia de Loja y al sur del Ecuador (Sudamérica) cuya superficie es de $1080,70 \mathrm{~km}^{2}$, siendo uno de los cantones más extensos de la provincia de Loja, con 30183 habitantes (SIN, 2015). La población de Saraguro está dividida en dos grupos étnicos identificados como: "indígenas" y "blancos", diferenciados por la vestimenta, costumbres y tradiciones (Deleg, 2010). Está a una altura de 2485 m s.n.m., presenta un clima templado andino, una temperatura media del aire de $17^{\circ} \mathrm{C}$ y una precipitación media de $600 \mathrm{~mm}$ (PDyOT Saraguro, 2015). Su topografía es montañosa, la influencia topográfica irregular da origen a una serie de pisos altitudinales y diversidad de suelos; la fertilidad del cantón es potencialmente alta (SIN, 2015). Los suelos presentan pH neutro a ligeramente alcalino; la disponibilidad de materia orgánica y nitrógeno es media, posee altos contenidos de potasio que son adecuados para la producción agrícola, mientras que el contenido de fósforo es bajo, lo cual repercute en la producción hortícola (Hernández, 2011).

La muestra de estudio constó de 40 participantes que forman parte del Consejo de Sanadores divididos de acuerdo con las actividades a las que se dedican. Unos 20 participantes (2 hombres y 18 mujeres) se dedican a la actividad de cultivar huertos "Grupo Huertos", los que cultivan y abastecen de materia prima (plantas medicinales y hortalizas) a los sanadores y mercado local. Por otra parte, otro grupo de 20 participantes (5 hombres y 15 mujeres), denominados "Grupo Sanadores", son los encargados de practicar la medicina ancestral (Armijos et al., 2014; Toledo et al., 2015). Cabe destacar en los dos grupos, la figura protagonista de las mujeres en el cultivo de huertos y como sanadoras y parteras, esto debido a que el hombre, al ser considerado jefe de familia, tiene un porcentaje de participación bajo y se atribuye, precisamente, a sus obligaciones centradas en buscar el sustento para su familia fuera del hogar (Jaramillo, 2016). 
Utilizando la herramienta FODA, se estableció el currículo de capacitación, se planificaron de manera participativa los temas a desarrollar y conforme a las necesidades del grupo (Pumisacho y Sherwood, 2005). El calendario de actividades se estableció acorde a la disponibilidad de tiempo de los participantes, la coordinación se realizó con el líder del grupo y los responsables del Programa Responsabilidad Social Universitaria con el Consejo de Sanadores Comunitarios de Saraguro. Las capacitaciones (Tabla 1) fueron dictadas en español, considerando la pérdida de la lengua materna Kichwa y situando al español como lengua predominante (Pérez, 1958).

Tabla 1. Currículo de capacitación para el manejo de huertos ecológicos en la comunidad Saraguro.

\begin{tabular}{|c|c|c|}
\hline $\mathbf{N}^{\circ}$ de taller & Tema & Metodología \\
\hline 1 & $\begin{array}{l}\text { - Definición e importancia de la agricultura } \\
\text { ecológica. } \\
\text { - Manejo del suelo. }\end{array}$ & $\begin{array}{l}\text { - Aplicación de encuesta inicial. } \\
\text { - Explicación teórica mediante diapositivas. } \\
\text { - Espacio de preguntas y respuestas. }\end{array}$ \\
\hline 2 & $\begin{array}{l}\text { - Implementación de un huerto familiar. } \\
\text { - Propagación (siembra directa e indirecta). } \\
\text { - Trasplante. }\end{array}$ & $\begin{array}{l}\text { - Explicación teórico-práctica. } \\
\text { - Análisis de pH del suelo. } \\
\text { - Espacio de preguntas y respuestas. } \\
\text { - Retroalimentación. }\end{array}$ \\
\hline 3 & $\begin{array}{l}\text { - Cuidados culturales (riego, aporque y } \\
\text { deshierbe). }\end{array}$ & $\begin{array}{l}\text { - Explicación teórico-práctica. } \\
\text { - Retroalimentación. } \\
\text { - Espacio de preguntas y respuestas. }\end{array}$ \\
\hline 4 & • Elaboración de abonos orgánicos. & $\begin{array}{l}\text { - Explicación teórico-práctica. } \\
\text { - Elaboración de abono líquido. } \\
\text { - Espacio de preguntas y respuestas. } \\
\text { - Retroalimentación. }\end{array}$ \\
\hline 5 & $\begin{array}{l}\text { - Manejo integrado de plagas y enfermedades. } \\
\text { - Manejo y cosecha de hortalizas. }\end{array}$ & $\begin{array}{l}\text { - Explicación teórica mediante diapositivas. } \\
\text { - Retroalimentación. } \\
\text { - Espacio de preguntas y respuestas. }\end{array}$ \\
\hline 6 & • Evaluación final. & $\begin{array}{l}\text { - Se culminó con la aplicación de una } \\
\text { encuesta final a los agricultores posterior a } \\
\text { las capacitaciones. }\end{array}$ \\
\hline
\end{tabular}

Fuente: elaboración propia.

Las capacitaciones se efectuaron aplicando la metodología de Escuela de Campo para Agricultores en el Desarrollo Rural (ECA) (Figura 1), la cual valora el conocimiento del productor y utiliza herramientas participativas que faciliten el aprendizaje de los participantes. Se emplearon herramientas como: presentaciones digitales, reuniones de grupo, estudios de caso de la comunidad, debates, análisis de experiencias y folletos.

Las capacitaciones desarrolladas a través de talleres teórico-prácticos fueron validadas por parte de los técnicos del proyecto y evaluadas por los participantes. 
Figura 1. Proceso de desarrollo de la escuela de campo, donde se integran los conocimientos locales y la información técnica del facilitador.

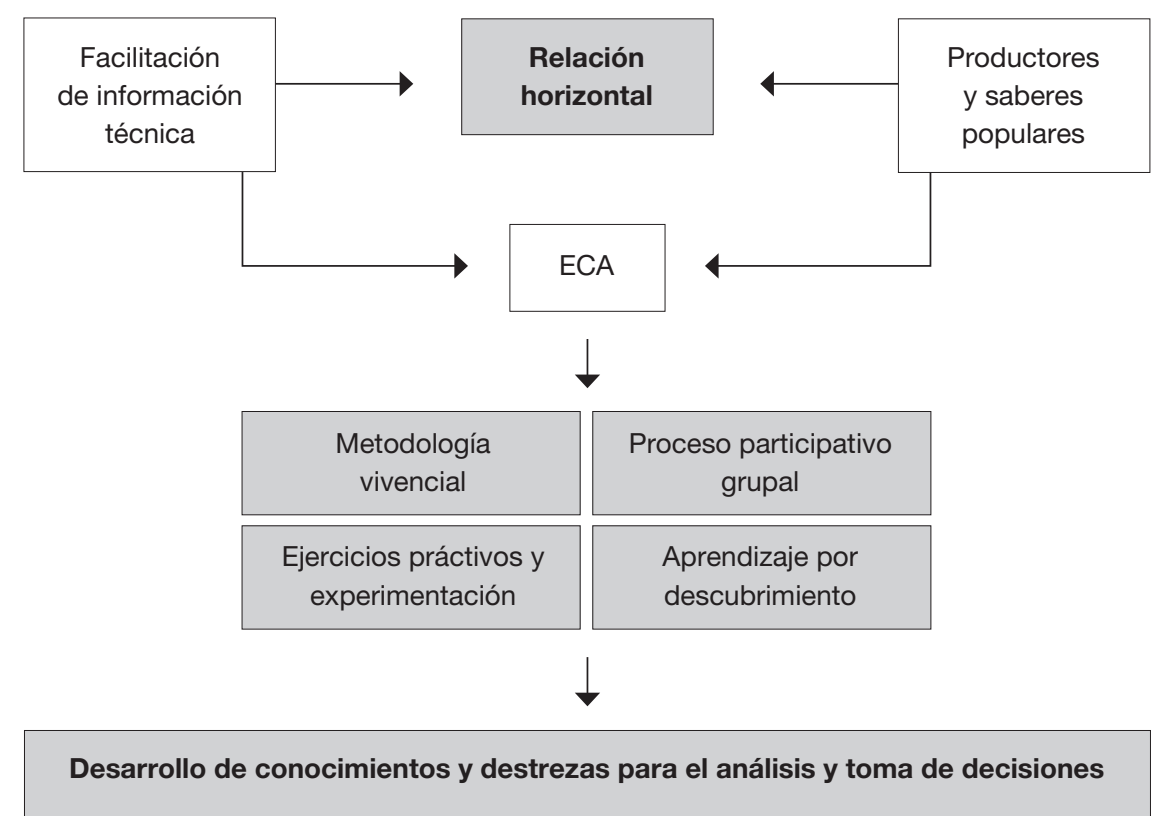

Fuente: Mejía (2003).

Para conocer el grado de aprendizaje de los participantes, y considerando su capacidad de lectura y escritura, se aplicó una encuesta dirigida por el equipo de trabajo para facilitar la comprensión de cada pregunta. Esto se realizó tanto al inicio como al final de las capacitaciones, según los temas del currículo definido. El modelo de encuesta utilizado fue diseñado conjuntamente por los técnicos atento a los temas que contempla el currículo de capacitación para el manejo de huertos familiares, y esas variables se tomaron de acuerdo con el análisis FODA y la información que se necesitaba recopilar. En cada taller de capacitación se registró la asistencia para conocer el grado de participación y el interés de los asistentes en los diferentes temas. Con el propósito de determinar el grado de aprendizaje de todos los temas, al final del programa de capacitación se hizo una evaluación para los participantes, la cual constó de un total de 25 preguntas de opción múltiple con tres distractores.

Los datos recopilados de las encuestas fueron codificados e ingresados a una base de datos para su análisis descriptivo. Para determinar las diferencias estadísticas significativas entre el conocimiento antes y después de las capacitaciones, se realizó una t de student con un nivel de significancia $p<0,05$.

\section{Resultados de la intervención}

Los resultados indican que, en la población muestreada (pobladores dedicados a actividades de producción en huertos y sanadores), más del $90 \%$ de los participantes se encuentra 
en una edad de entre los 34 y 65 años. Por otra parte, se evidenció que en el grupo de "sanadores" no hubo ningún participante menor de 34 años; la participación de personas jóvenes en este grupo posiblemente no se dio debido a que, para realizar ese tipo de actividades de sanación, se deben tener conocimientos básicos y además probarlos, lo cual solo sucede con la experiencia y el tiempo, por lo que esa actividad en esta comunidad recae únicamente en personas de adultas a ancianas. En tanto, en el grupo de los "Productores de huertos" sí hubo personas menores de 34 años (10\%).

La baja participación de personas jóvenes en actividades de carácter agropecuario se dio mayormente por la migración alta, la cual por lo general es a zonas urbanas del Ecuador, y esto por la búsqueda de otro tipo de trabajo (diferente de la agricultura), por estudios, o simplemente por falta de interés en involucrarse en el sector agropecuario. Además, muchas personas consideran que este tipo de actividades son de un bajo estatus social y mal remuneradas. Por otra parte, muchos de los habitantes de esta zona han migrado hacia otros países con el fin de mejorar su economía y la de su familia (Banco Central del Ecuador, 2018). Cabe indicar que el abandono de las actividades agrícolas y la baja remuneración en esta actividad están afectando en un grado considerable a la seguridad alimentaria de sus habitantes, ya que en la actualidad los alimentos que antes producían ahora deben ser adquiridos, y en muchos de los casos la economía no es lo suficientemente estable como para adquirir todo lo necesario (FAO, 2018).

En lo que respecta a las capacitaciones, se debe indicar que la asistencia y el desenvolvimiento de los agricultores para llevar a cabo la encuesta, tanto al inicio como al final, fueron muy buenos, lo que denota la predisposición y colaboración en la ejecución del proyecto. La asistencia del total de participantes fue del $90 \%$ en todos los talleres impartidos, y el $10 \%$ restante no asistió debido a sus ocupaciones en el campo. No obstante, cabe destacar que el compromiso de los participantes siempre fue activo y responsable.

\section{Desinfección del suelo}

En el manejo y producción de cultivos, sean de ciclo corto o perennes, la desinfección del suelo es uno de los primeros pasos a realizar y por eso fue el primer tema en las capacitaciones. Al hacer la primera encuesta, se pudo apreciar que las personas participantes que tenían este conocimiento y ya desinfectaban el suelo era aproximadamente del $50 \%$. Estos grupos mencionaron que este tipo de desinfección lo hacían con cal y ceniza, a la vez que comentaron que la desinfección del suelo con estos materiales les servía como fuente de materia orgánica y nutrientes, lo que les ayudaba a mejorar significativamente los rendimientos de sus cultivos (Awodun, 2007). En menor grado, la cal también es utilizada puesto que ayuda a reducir los hongos y patógenos del suelo y, además, contribuye a reducir la acidez del suelo e incrementa el calcio y magnesio (Osorno y Zapata, 2012). El otro $35 \%$ de los participantes, pese a conocer acerca de las actividades de desinfección del suelo, no realizaba esta actividad porque no la consideraba relevante en los procesos productivos.

Con posterioridad a las capacitaciones, el nivel de conocimiento sobre la desinfección del suelo y su importancia subió al $80 \%$ en los dos grupos (Grupo de Huertos y Sanadores), y este porcentaje final comprendió la importancia y beneficios de la desinfección del suelo $y$, por ende, se comprometió a efectuar esta práctica. Este valor resulta ser un porcentaje importante que nos indica que, a pesar de que partimos de un conocimiento y experiencia 
previos, se debe fortalecer y llegar al resto del grupo para difundir la información adquirida a un grupo más amplio. También se les compartió información sobre productos comerciales para esta actividad. Sin embargo, dados los costos, los participantes en su mayoría optarían por los mismos materiales que siempre tuvieron disponibles (cal y ceniza).

\section{Elaboración de abonos orgánicos}

En los dos grupos se advirtió que los participantes tenían conocimientos sobre la elaboración de los abonos orgánicos, e indicaron los hacían y aplicaban el $73 \%$ de ellos. Los principales abonos usados son el compost y el humus debido a que se conoce el procedimiento para la elaboración de los mismos. Esa preparación se hace a base de los residuos de cocina, del sobrante de las cosechas y de excretas de animales. Por otra parte, también fabrican abonos líquidos como el biol y el té de estiércol, aunque este último lo hace un menor porcentaje (Tabla 2) porque muchos de ellos no conocen el proceso para su preparación y aplicación.

Después de impartir los talleres de esta temática, el porcentaje de participantes con este conocimiento en la elaboración de abonos orgánicos subió a alrededor de un $96 \%$, lo cual indica que, con los conocimientos técnicos adquiridos en los talleres, poseen la capacidad de elaborar este tipo de abonos y de una manera más adecuada a como lo hacían antes. Además, comentan que los beneficios de su aplicación son muy importantes ya que así mejoran las propiedades edáficas, incrementando la fertilidad del suelo y, por lo tanto, eso beneficia la producción de sus cultivos. En cuanto a la elaboración de biol dentro del grupo de capacitados, tuvo una aceptación muy buena, puesto que aumentó a más del doble su conocimiento entre los participantes. Sin embargo, en el caso del té de estiércol, este porcentaje se incrementó aproximadamente solo en un $23 \%$ desde antes de las capacitaciones hasta el final de las mismas, siendo solo la mitad de los capacitados (20 participantes) los interesados en desarrollar este tipo de fertilizante o abono (Tabla 2) debido a que consideraron que con la elaboración del biol es suficiente para aplicar a los cultivos.

El reciclaje de los desechos orgánicos trae consigo muchas ventajas, por ejemplo, su elaboración y aplicación en las fincas o espacios de producción de cultivos disminuye los costos en la adquisición de abonos y fertilizantes y por lo tanto sus costos de producción. Asimismo, contribuye a la fertilidad y conservación del recurso suelo, haciendo más sustentable su producción y fincas (Clavijo y Cuvi, 2017). Cabe recalcar que el abono orgánico a preparar y aplicar al suelo dependerá de los materiales que se encuentren disponibles en la zona y específicamente en su finca, razón por la cual en las capacitaciones teórico-prácticas se impartieron conocimientos con varias opciones de materiales para realizar los abonos, a fin de que el productor seleccione el más viable según sus condiciones.

\section{Plagas, enfermedades y biopreparados}

Uno de los mayores problemas presentes en la zona, y que limita de manera considerable las cosechas, es la presencia de plagas y enfermedades. Los errores en su identificación llevan a un incorrecto control y manejo de las mismas, lo que causa problemas con la productividad de los cultivos. El conocimiento de los participantes antes del desarrollo de las capacitaciones en el tema de la clasificación, daño e identificación de plagas y enfermedades que afectan a sus cultivos, fue considerable, con alrededor del $50 \%$ para los dos grupos y, luego de la capacitación, su conocimiento aumentó en un $36 \%$ (conocimiento final del 86 \% en los 
dos grupos) (Tabla 2). Algunos de los participantes, antes de nuestra intervención, ya habían recibido capacitación en el tema de plagas y enfermedades, sin embargo, ellos indicaban que aún deseaban un mayor desarrollo en sus conocimientos en este tema debido a que era uno de los problemas que causaba mayores daños y pérdidas a la hora de producir, por lo cual los dos grupos capacitados presentaron muy buena aceptación al plan de capacitación e incluso atrajeron a otros participantes del sector. Al final de estas capacitaciones, indicaron que el conocimiento sobre el control y manejo de plagas y enfermedades les ayudó a mejorar la producción y la calidad de las hortalizas, potencializando los huertos familiares, ya que lograron adaptar las técnicas aprendidas al manejo integrado de plagas y enfermedades y minimizaron la utilización de productos químicos.

Tabla 2. Resumen general de la evaluación de conocimiento inicial (antes de la capacitación) y final (después de la capacitación).

\begin{tabular}{|c|c|c|}
\hline Contenido & $\%$ Antes & $\%$ Después \\
\hline \multicolumn{3}{|l|}{ Abonos orgánicos } \\
\hline Conoce los beneficios & 73,12 & 96,25 \\
\hline \multicolumn{3}{|l|}{ Abonos sólidos } \\
\hline Humus & 47,50 & 79,37 \\
\hline Compost & 67,50 & 71,80 \\
\hline \multicolumn{3}{|l|}{ Abonos líquidos } \\
\hline Biol & 42,50 & 92,50 \\
\hline Té de estiércol & 27,50 & 50,60 \\
\hline \multicolumn{3}{|l|}{ Plagas y enfermedades } \\
\hline Conocimiento y clasificación de plagas y enfermedades & 52,50 & 88,75 \\
\hline Daño que ocasionan al cultivo & 65,00 & 72,00 \\
\hline \multicolumn{3}{|l|}{ Control de plagas y enfermedades } \\
\hline Conocimiento de briopreparados & 49,00 & 99,37 \\
\hline
\end{tabular}

Fuente: elaboración propia.

En cuanto a la elaboración y aplicación de los biopreparados, los participantes de los dos grupos, al inicio de las capacitaciones, tenían un conocimiento menor al $50 \%$. Después de la capacitación sobre esta temática, los resultados fueron muy alentadores, el conocimiento en el tema llegó por poco al 100 \% (Tabla 2), es decir que los encuestados adquirieron los conocimientos básicos para la preparación y aplicación de los biopreparados en los cultivos, 
puesto que su aplicación cumple con el objetivo de corregir los desequilibrios ocasionados por el ataque de plagas y enfermedades. Los biopreparados permiten llevar una agricultura sostenible, sea a pequeña o a gran escala, gracias a su facilidad de preparación y aplicación, además de su bajo costo, ya que los materiales que se utilizan se encuentran en las comunidades y son de rápida degradación, lo que significa menor riesgo de contaminación al ambiente (FAOb, 2010).

\section{Fortalezas y debilidades de la comunidad}

Las reuniones de capacitación reforzaron la organización comunitaria, además del compromiso y puntualidad del grupo. Por otra parte, ayudaron a corregir de manera significativa las diferencias intergrupo, principalmente para los "Sanadores", que son quienes tenían menos cultivos implementados, por lo que las temáticas impartidas eran de su total interés. Mientras tanto, para el grupo de las personas dedicadas a los "Huertos", los porcentajes de organización y puntualidad en ciertos temas disminuyeron, lo cual se atribuyó a que ese grupo ya tenía instalados sus huertos y, en algunos casos, poseían un mayor conocimiento de su manejo.

En general, las capacitaciones posibilitaron que los dos grupos mejoraran sus conocimientos para ser proveedores de su propia materia prima, fomentaron la integración de la comunidad y la generación de nuevas cualidades y competencias, lo que les permitirá, a su vez, desarrollarse conjuntamente a través de la transferencia de conocimientos adquiridos hacia sus familiares, amigos y comunidad, con un impacto positivo a nivel comunitario.

\section{Transferencia de conocimientos a los participantes}

Para medir el grado de conocimientos de los participantes se realizó una evaluación escrita antes y después de las capacitaciones. Asimismo, los docentes y estudiantes evaluaron de manera observacional el desarrollo práctico por parte de los participantes. En la Figura 2 se puede apreciar que las capacitaciones fueron eficientes, se advierte que el promedio general de conocimientos y capacidades de los participantes aumentó en un total de 6 puntos, lo cual genera un impacto positivo para toda su comunidad. En tanto, al realizar la consulta de satisfacción a los participantes, un $90 \%$ indicó que esta fue muy satisfactoria, puesto que los dos grupos (Sanadores y Huertos) comentaron que poseen capacidad para manejar sus cultivos y que están seguros de lograr mejorar la productividad. Por otra parte, también indicaron que tienen la capacidad de difundir y enseñar los conocimientos adquiridos, ya sea a los miembros de sus familias como a la comunidad que lo requiera.

\section{Experiencia de los capacitadores}

Los talleres de capacitaciones no solo dejaron enseñanzas al participante, sino que también contribuyeron a que los capacitadores (docentes y estudiantes) comprendieran con mayor detalle los problemas y necesidades de la comunidad y en función de ello puedan trabajar de manera específica en uno o varios temas. También aportaron a la mejora en sus conocimientos personales y profesionales y generaron mayor motivación para la participación en estas experiencias o proyectos de vinculación con las comunidades rurales indígenas. Finalmente, cabe señalar que han sido muy satisfactorios, lo cual es esencial para plantear e implementar otros talleres formativos en beneficio de estos sectores. 
Figura 2. Promedio de la evolución del conocimiento antes y después de las capacitaciones (evaluación sobre 20 puntos).

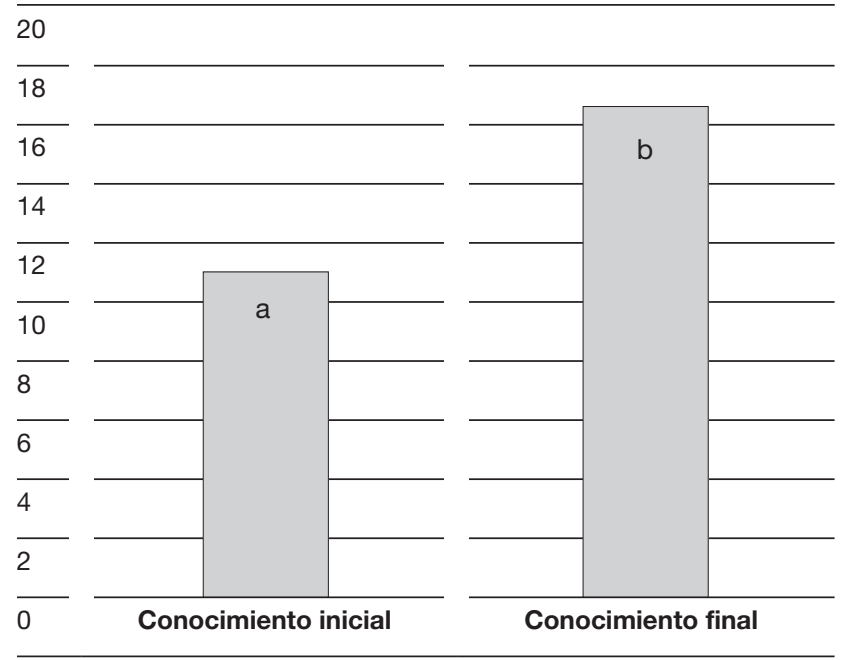

Las barras muestran el conocimiento promedio inicial y final de 40 participantes. Las letras minúsculas muestran diferencias estadísticas significativas $(p<0,05)$

Fuente: elaboración propia.

\section{Conclusiones}

Al ser las actividades agrícolas la principal fuente de manutención de los miembros del Consejo de Sanadores Comunitarios de la etnia Saraguro, las capacitaciones enfocadas a estas temáticas agroproductivas sostenibles son unas de las estrategias más viables para mejorar la producción de sus huertos y cultivos, y en este caso se logró contribuir a la mejora de conocimientos y capacidades en los participantes y además benefició a su seguridad alimentaria. Es oportuno indicar que estos resultados son alentadores porque demuestran el interés de los grupos encuestados por aprender y mejorar las técnicas de producción.

Por otra parte, la enseñanza dejada a los capacitadores (docentes y estudiantes) es muy satisfactoria, ya que se vivieron las problemáticas y necesidades que afronta este tipo de comunidades, lo cual produjo un impacto positivo, y de manera especial en los estudiantes, porque se dieron cuenta que la extensión comunitaria, además de las enseñanzas profesionales, deja enseñanzas gratificantes en cuanto a la convivencia, el trabajo en equipo y la colaboración.

Los resultados son alentadores porque demuestran el interés de los grupos por aprender y mejorar la producción a pesar del poco tiempo de capacitación.

\section{Referencias bibliográficas}

Andrade, J.M.; Armijos, C.; Malagón, O.; Lucero, H. (2009). Plantas silvestres empleadas por la etnia Saraguro en la Parroquia San Lucas. Loja: Universidad Técnica Particular de Loja.

Armijos, C.; Cota, L.; González, S. (2014). Traditional medicine applied by the Saraguro yachakkuna: a prelimi- 
nary approach to the use of sacred and psychoactive plant species in the southern region of Ecuador. Journal of Ethnobiology and Ethnomedicine, 10(26), 1-12.

Awodun, M.A. (2007). Effect of Sawdust Ash on Nutrient Status, Growth and Yield of Cowpea (Vigna unguiculata L. Walp). Asian Journal of Agricultural Research, 1(2), 92-96.

Banco Central del Ecuador (2018). Evolución de las remesas región Austro, 2do. trimestre. Recuperado de https://contenido.bce.fin.ec/frame.php?CNT=ARB0000985 (30/05/2018).

Clavijo, C. y Cuvi, N. (2017). La sustentabilidad de las huertas urbanas y periurbanas con base agroecológica: el caso de Quito. Revista Latinoamericana de Estudios Socioambientales, (21), 68-91.

Cueva, J. y Chalán, L. (2010). Cobertura vegetal y uso actual del suelo de la Provincia de Loja. Informe Técnico Departamento de Sistemas de Información Geográfica de Naturaleza y Cultura Internacional.

Deleg, G. y Zhunaula, S. (2010). Análisis microeconómico de los hábitos de consumo de las familias indígenas y mestizas de la cabecera cantonal de Saraguro (Tesis de pregrado). Universidad de Cuenca, Ecuador.

Encuesta de Superficie y Producción Agropecuaria Continua (ESPAC) (2015). Presentación de resultados ESPAC. En Instituto nacional de Estadística y Censo, Ecuador.

Hernández, F. (2011). Preparación de tierra para siembra de hortalizas. Recuperado de http://www.agro-tecnologia-tropical.com/preparacion_suelos.html

Instituto Interamericano de Cooperación para la Agricultura (IICA) (1992). Capacitación campesina con metodologías participativas en la región Andina. Ibagué, Colombia.

Instituto Nacional de Estadística y Censo del Ecuador (2015). Encuesta de superficie y producción agropecuaria continua representación de resultados ESPAC.

Jaramillo, A. (2016). Caracterización de cadenas productivas en los productos elaborados por el Consejo de Sanadores del cantón Saraguro.

López, J.; Jiménez, L.; León, A.; Figueroa, O.; Morales, M.; González, V. (2008). Escuelas de campo, para capacitación y divulgación con tecnologías sustentables en comunidades indígenas. Agricultura Técnica en México, 34(1), 33-42.

Marín I.; Hinojosa M.; López A.; Carpio L (2016). El San Pedro y la mujer Saraguro. La medicina tradicional aplicada por la yachakkuna. VII Congreso virtual sobre Historia de Las Mujeres 433-446 del 15 al 31 de octubre. Marín, J. (2017, 14 de enero). La medicina ancestral convive con la clínica. Diario el Expreso.

Mejía, A. (2003). Las Escuelas de Campo para Agricultores en el Desarrollo Rural. MAGAP. SIN, Quito, Ecuador. Recuperado de http://www.fao.org/climatechange/30315069f5a40da3e46706f6936d2e99514e30.pdf (30/05/2018).

Ministerio de Salud (2016). Alimentación Saludable. Recuperado de http://www.fao.org/docrep/014/am401s/ am401s02.pdf (30/05/2018).

Monteros, G. y Salvador, S. (2015). Panorama agroeconómico del Ecuador una visión del 2015. Dirección de Análisis y Procesamiento de la Información, Coordinación General del Sistema de Información Nacional, Ministerio de Agricultura, Ganadería, Acuacultura y Pesca.

Núñez, J. (2004). Los saberes campesinos: implicaciones para una educación rural. Investigación y Postgrado, 19(2), 11-58.

Organización de las Naciones Unidas para la Agricultura y la Alimentación (FAO) (2010). Cómo movilizar el potencial de la extensión agraria y rural. Recuperado de http://www.fao.org/docrep/013/i1444s/i1444s00. pdf (30/05/2018).

(2018). Seguridad alimentaria y nutricional en América Latina y el Caribe. http://www.fao.org/americas/ prioridades/seguridad-alimentaria/es/ (30/05/2018). 
Organización de las Naciones Unidas para la Agricultura y la Alimentación (FAO), Promoción del Desarrollo Sostenible (IPES) (2010). Biopreparados para el manejo sostenible de plagas y enfermedades en la agricultura urbana y periurbana. IPES/FAO.

Organización Mundial de la Salud (OMS) (2013). Estrategia de la OMS sobre medicina tradicional 2014-2023. Recuperado de http://apps.who.int/medicinedocs/documents/s21201es/s21201es.pdf (30/05/2018).

Osorno, H. y Zapata, R. (2012). Mitos y realidades de las cales y enmiendas en Colombia. Universidad de Colombia. Tesis de doctorado.

Pérez, A.R. (1958). Contribución al conocimiento de la prehistoria de los pueblos del norte del territorio de la república del Ecuador. Instituto Ecuatoriano de Antropología y Geografía.

Pumisacho, M. y Sherwood, S. (2005). Guía Metodológica sobre Escuelas de Campo de Agricultores. Quito: CIP - INIAP - World Neighbors.

Sistema Nacional de Información (SIN) (2015). Recuperado de http://app.sni.gob.ec/visorseguimiento/ DescargaGAD/data/sigadplusdiagnostico/1160001130001_DIAGNOSTICO-\%20PDyOT\%20Saraguro2015_15-03-2015_19-08-17.pdf (30/05/2018).

Suárez, G. (2000). Los extensionistas agrícolas como promotores del desarrollo comunitario. Ministerio de Agricultura y Ganadería de Costa Rica. Recuperado de http://www. mag. go.cr/docc/004.html (30/05/2018). 